\title{
Ultrasound Modulates Fluorescence Strength and ABCG2 mRNA Response to Aminolevulinic Acid in Glioma Cells
}

\author{
Tadashi Higuchi ${ }^{1}$, Fumio Yamaguchi ${ }^{2}$, Takayuki Asakura ${ }^{1}$, \\ Daizo Yoshida ${ }^{1,3}$, Yumiko Oishi ${ }^{4}$ and Akio Morita ${ }^{1}$ \\ ${ }^{1}$ Department of Neurological Surgery, Nippon Medical School, Tokyo, Japan \\ ${ }^{2}$ Department of Neurosurgery for Community Health, Nippon Medical School Graduate School, Tokyo, Japan \\ ${ }^{3}$ Rihabilipark Itabashi Hospital, Tokyo, Japan \\ ${ }^{4}$ Department of Biochemistry \& Molecular Biology, Nippon Medical School, Tokyo, Japan
}

\begin{abstract}
Background: 5-Aminolevulinic Acid (5-ALA) photodiagnosis (PD) is an effective method to detect residual tumors during glioma surgery. However, fluorescence strength differs in malignant gliomas, and false-negative fluorescence may result in tumor residue. We investigated the effect of ultrasound on the intracellular level of protoporphyrin IX (PpIX) and expression level of ATP-binding cassette transporter 2 (ABCG2), which is thought to act as a membrane efflux pump of PpIX from cytosol.

Methods: The malignant glioma cell lines SNB19, U87MG, and T98G were used for in vitro experiments. Cultured cells underwent ultrasound irradiation $\left(1 \mathrm{MHz}, 3 \mathrm{~W} / \mathrm{cm}^{2}\right.$, duty cycle $\left.10 \%\right)$ after administration of 5-ALA, and morphological changes in tumor cells were observed. PpIX levels and ABCG2 expression were evaluated.

Results: The glioma tumor cells showed transient morphological changes and detachment from the culture dish; however, most cells survived and reverted to their original morphology within 6 hours. PpIX expression levels increased in glioma cells after ultrasound irradiation, and the increase was earlier and greater than that for 5-ALA alone. ABCG2 expressions increased after 5-ALA administration but were lower in ultrasound-irradiated glioma cells.

Conclusions: Ultrasound irradiation of malignant gliomas contributes to stronger 5-ALA-induced fluorescence by elevating intracellular PpIX levels. Suppression of ABCG2 expression by ultrasound may contribute to PpIX accumulation in glioma cells. (J Nippon Med Sch 2020; 87: 310-317)
\end{abstract}

Key words: 5-ALA, protoporphyrin IX, ABCG2, malignant glioma, ultrasound

\section{Introduction}

Photodiagnosis (PD) of brain tumors is widely used to detect tumors during surgery, and the photosensitizer 5aminolevulinic acid (5-ALA) is available in many countries for intraoperative diagnosis of malignant glioma.

The strength of fluorescence differs in relation to histology, malignancy, timing of 5-ALA administration, and multiple other factors. When 5-ALA is used clinically for detecting malignant brain tumors, the variability of fluorescence impairs its reliability for diagnosis. The efficiency of PD depends on the level of protoporphyrin IX
(PpIX) accumulated in tumor cells in the relevant target tissue. Therefore, PpIX accumulation must be enhanced in targeted tumor cells for stable detection.

Exogenous 5-ALA is incorporated into cells via transporters such as peptide transporter 1 (PEPT1) and peptide transporter 2 (PEPT2). Expression levels of these transporters affect the amount of 5-ALA incorporated, thus altering the strength of fluorescence ${ }^{1}$. In the subsequent porphyrin synthetic pathways, ferrochelatase $(\mathrm{FECH})$, an enzyme that catalyzes chelation of ferrous iron to PpIX to form heme, is thought to be a factor that

Correspondence to Fumio Yamaguchi, Department of Neurosurgery for Community Health, Nippon Medical School Graduate School, 1-1-5 Sendagi, Bunkyo-ku, Tokyo 113-8603, Japan

E-mail: fyamaguc@nms.ac.jp

https://doi.org/10.1272/jnms.JNMS.2020_87-601

Journal Website (https://www.nms.ac.jp/sh/jnms/) 


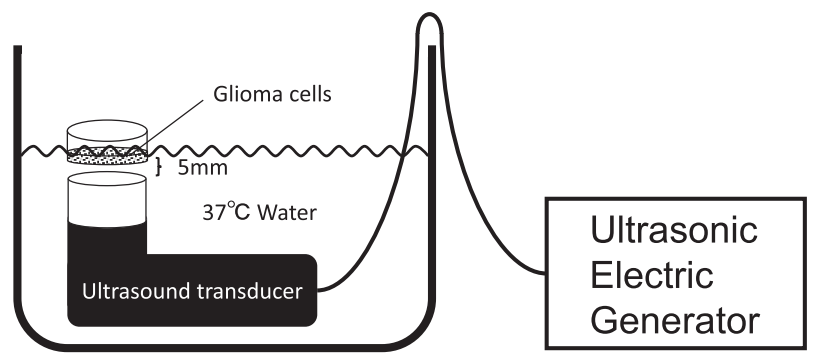

Fig. 1 Ultrasound irradiation system The ultrasound device used in this study comprised an ultrasonic electronic generator, amplifier, and transducer and was manufactured by Ito CO., LTD (Tokyo, Japan). The ultrasound transducer was fixed at the bottom of a thermostatic $37^{\circ} \mathrm{C}$ water bath. The cell culture dish was suspended $5 \mathrm{~mm}$ above the ultrasound transducer.

affects PpIX accumulation in cytosol ${ }^{1}$. Ultimately, accumulated PpIX is excreted by the membrane transporter ATP-binding cassette $(\mathrm{ABC})$ transporter 2 (ABCG2) on the cell membrane. Human ABC transporter ABCG2, originally called breast cancer-resistant protein (BCRP), was first discovered in doxorubicin-resistant breast cancer cells. Recently ABCG2 was reported to be present in the plasma membrane as a homodimer bound through disulfide-bonded cysteine residues ${ }^{3-5}$. This transporter may be important in regulating intracellular porphyrin ${ }^{6-10}$.

Ultrasound induces biological modulations, including intensification or inactivation of enzyme activity ${ }^{11}$ and inhibition of atherosclerotic plaque progression ${ }^{12}$. A common therapeutic application, ultrasound is used in many types of sonodynamic therapy, which utilizes various sonosensitizers. The mechanism is based on activation of these sonosensitizers, a process that generates free radicals such as singlet oxygen species. Ultrasound was recently reported to reverse chemoresistance in breast cancer stem-like cells by altering ABCG2 expression?. Therefore, it is worthwhile to investigate its effect in glioma cells.

This study examined the effects of ultrasound on PpIX expression in malignant glioma cells after 5-ALA administration and the mechanisms underlying these effects. Our principal finding was that early and increased accumulation of PpIX in glioma cells after ultrasound was accompanied by ABCG2 downregulation.

\section{Materials and Methods \\ Chemicals and Reagents}

5-ALA purchased from Cosmo Bio Co., Ltd. (Tokyo, Japan) was dissolved in a 1 N HCL solution to make a 100 $\mathrm{mM}$ stock solution that was sterilized, aliquoted, and stored at $-80^{\circ} \mathrm{C}$ in the dark. Dulbecco's modified Eagle's medium (DMEM), fetal bovine serum (FBS), streptomycin, and penicillin were obtained from Gibco (Grand Island, NY, USA).

\section{Cell Lines and Cell Culture}

SNB19 is a well-characterized human glioblastoma cell line $^{13}$. U87MG and T98G, the other human primary glioblastoma cell lines, were purchased from ATCC (American Type Culture Collection, Manassas, VA, USA). The human glioma cell lines SNB19, U87MG, and T98G were cultured in DMEM containing 10\% FBS, $100 \mathrm{U} / \mathrm{mL}$ penicillin, and $100 \mu \mathrm{g} / \mathrm{mL}$ streptomycin at $37^{\circ} \mathrm{C}$ in a humidified incubator in an atmosphere of $5 \% \mathrm{CO}_{2}$. The cells were harvested for passage twice per week when they were nearly confluent, and $4 \times 10^{5}$ cells were seeded in a 35-mm culture dish and incubated for 24 hours. 5-ALA was added at a final concentration of $1.0 \mathrm{mM}$. Then, the cells were used in the following experiments.

\section{Ultrasound Device and Conditions}

The ultrasound device used in this study comprised an ultrasonic electronic generator, amplifier, and transducer and was manufactured by Ito CO., LTD (Tokyo, Japan). The ultrasound transducer (diameter: $3.5 \mathrm{~cm}$, resonance frequency: $1.0 \mathrm{MHz}$, duty factor: $10 \%$, ultrasonic intensity: $3.0 \mathrm{~W} / \mathrm{cm}^{2}$ ) was fixed at the bottom of a thermostatic water bath. The cell culture plate was suspended 5 $\mathrm{mm}$ above the ultrasound transducer, as shown in Figure 1. The temperature of the water bath was $37^{\circ} \mathrm{C}$. The whole experiment was conducted in the dark to avoid a photodynamic therapy effect. Then, the cells were incubated and collected separately at $0,1,2,3,4,5$, and 6 hours after adding 5-ALA. The duration of observation was based on the dynamics of absorption, metabolism, and excretion of 5-ALA in human glioma cells ${ }^{14}$. Two groups were studied-an ALA and ALA+US group. Cells in the ALA+US group were irradiated for 2 minutes by ultrasound under the above conditions 5 minutes after ALA exposure at a concentration of $1.0 \mathrm{mM}$.

Observation of Morphological Change of Tumor Cells

Morphological changes in treated tumor cells were observed with a microscope (TS100, Nikon, Tokyo, Japan), and the images were recorded with a digital recording system (DS-L1-5M, Nikon, Tokyo, Japan).

\section{Gaging of PpIX Accumulation in Glioma}

The treated cells were washed twice with PBS, and the collected cells were then centrifuged into pellets. Then, the fluorescence spectrum of PpIX was immediately detected with a spectrometer with 405-nm excitation and 
390- to 800-nm emission (VLD-EX, SBI Pharmaceuticals Co., Ltd., Tokyo, Japan). A sharp peak representing the strength of fluorescence was detected at an emission of $636 \mathrm{~nm}$. Intensity data were analyzed with Spectrometer Data Acquisition Software (BWSpec 3.26_14, Newark, DE, USA). All experiments were performed under dim light.

\section{mRNA Extraction}

Total RNA was extracted from glioma cells in each condition by using an RNeasy Mini Kit in accordance with the instructions of the manufacturer (QIAGEN, Hilden, Germany). Each RNA sample was quantified by NanoDrop (Fisher Scientific, Altham, MA, USA). cDNA synthesis was done with $1 \mu \mathrm{g}$ total RNA by using a PrimeScript RT Reagent Kit (Perfect Real Time), in which oligo dT primer and random 6mers were used in accordance with the instructions of the manufacturer (TaKaRa, Shiga, Japan), followed by reverse transcription for 15 minutes at $37^{\circ} \mathrm{C}$ and enzyme inactivation for 5 seconds at $85^{\circ} \mathrm{C}$.

\section{Quantitative Real-Time PCR}

Quantitative Real-Time PCR (qRT-PCR) was performed by using a StepOnePlus ${ }^{\text {TM }}$ Realtime PCR system (Thermo Fisher Scientific, Waltham, MA, USA) in accordance with the manufacturer's protocol. The ABCG2 probe was designed by the manufacturer (Gene Name: ATP-binding cassette subfamily G member 2 [Junior blood group], Assay ID: Hs01053790_m1) (TaqMan Gene expression assays; Thermo Fisher Scientific). GAPDH was used for internal normalization, and the probe was designed by the manufacturer (glyceraldehyde-3-phosphate dehydrogenase, Assay ID: Hs02786624_g1). Thermal cycling was performed under the following conditions: 40 cycles of $95^{\circ} \mathrm{C}$ for 1 second and $60^{\circ} \mathrm{C}$ for 20 seconds. Each experiment was performed in triplicate. Relative gene expression was analyzed by the $2-\Delta \Delta \mathrm{Ct}$ method. The expression levels of target genes were normalized against those of GAPDH by using StepOne ${ }^{\text {TM }}$ Software v2.3 (Thermo Fisher Scientific, Inc. Waltham, MA, USA). Relative quantity values were standardized by setting the same threshold value. Gene expression data are presented as mean \pm standard error of the mean of the RQ values.

\section{Results}

\section{Morphological Changes in Treated Tumor Cells}

The three glioma cell lines used in this study have a fusiform morphology, resembling a fibroblast. ALA administration caused minimal changes to this form. Ultrasound irradiation plus ALA administration shortened the spike of the cells, which tended to become small and round. In U87MG cells, some attached glioma cells detached from the floor of the culture dish after ultrasound. However, these cells reattached to the floor of the dish and reverted to the original shape of glioma cells (Fig. 2). Morphological changes in these cells observed immediately after ultrasound irradiation reverted to their pretreatment state within 6 hours.

\section{Intracellular Accumulation of PpIX}

Spectrometry confirmed intracellular accumulation of PpIX in glioma cell lines. A sharp peak at $636 \mathrm{~nm}$, indicating characteristic PpIX fluorescence spectra, was detected in SNB19, U87MG, and T98G cells (Fig. 3). As we previously reported ${ }^{15}$, there was time-dependent intracellular accumulation of PpIX in glioma cell lines exposed to exogenous 5-ALA. The level of PpIX accumulation differed in relation to cell line.

The relative intensity of PpIX in the ultrasoundirradiated group peaked at 4 hours after ultrasound exposure in the SNB19 and U87MG cell lines (Fig. 4), while PpIX expression in T98G continued to increase until 6 hours after ultrasound exposure. In all cell lines, the increase in PpIX expression was greater than that in the 5ALA only group at all time points, except at 6 hours in SNB19 cells (Fig. 4).

mRNA Expression of Membrane Transporter ABCG2 after 5-ALA Administration

Relative mRNA expression levels of ABCG2 were analyzed by qRT-PCR in each cell line. 5-ALA administration induced gradual elevation of ABCG2 expression in SNB 19 and U87MG cells. However, T98G cells did not exhibit a significant change after 5-ALA administration (Fig. 5).

Effect of Ultrasound Irradiation on ABCG2 mRNA Expressions

Ultrasound irradiation suppressed the increase in ABCG2 expression induced by ALA exposure (Fig. 5). This effect was seen in all three cell lines and persisted for 2 hours after ultrasound irradiation in U87MG and T 98G cells. Within 5 hours after ultrasound irradiation, this effect gradually diminished, and the ABCG2 mRNA level increased to the level of the non-ultrasound condition in T98G cells. In U87MG cells, ABCG2 expressions were higher than in the ALA only group. However, this suppression effect persisted for 6 hours in SNB19 cells.

\section{Discussion}

\section{Cell Viability by Ultrasound Irradiation}

Microscopy showed transient morphological changes and loss of cell adhesion to the floor of the culture dish. 


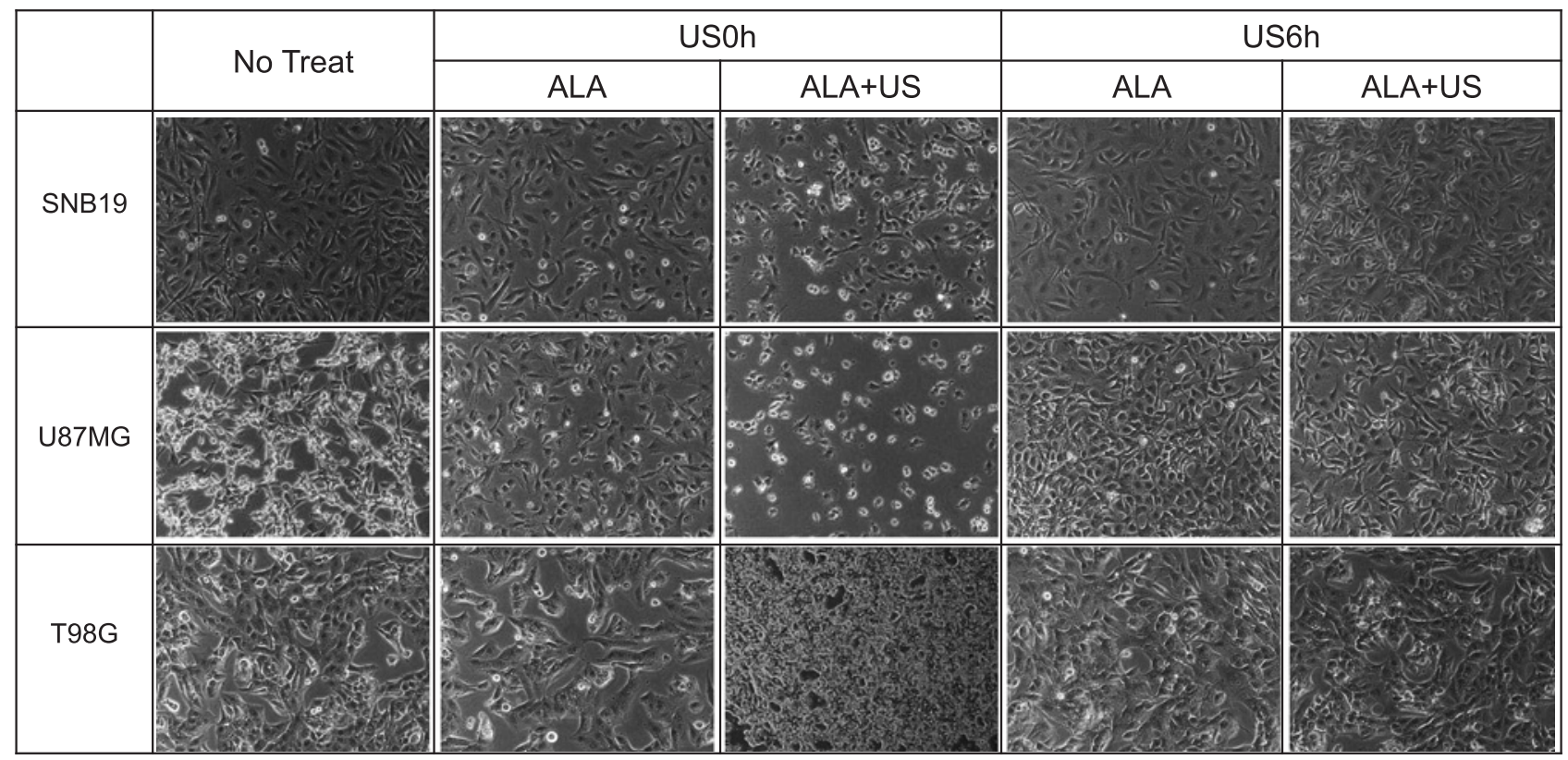

Fig. 2 Morphological changes of treated tumor cells

The three glioma cell lines showed minimal morphological changes after ALA administration. Ultrasound irradiation plus ALA administration shortened the spike of cells, which tended to become small and round. In U87MG cells, some attached glioma cells detached from the floor of the culture dish after ultrasound. However, these cells reattached to the floor of the dish and reverted to the original shape of glioma cells within 6 hours after treatment. Morphological changes of cells immediately after ultrasound irradiation reverted to the pretreatment state within 6 hours (US0h; immediately after ultrasound irradiation, US6h; 6 hours after ultrasound irradiation, ALA; only ALA administered, ALA+US; ALA administration followed by ultrasound irradiation).

SNB19
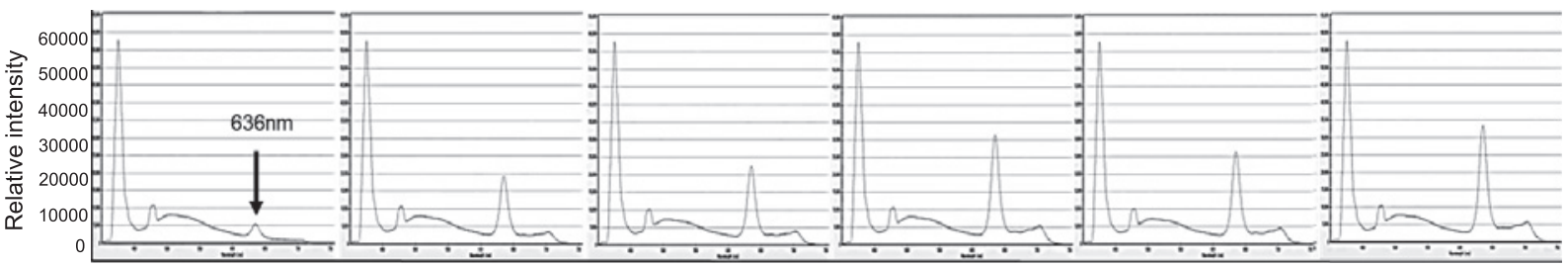

Wavelength
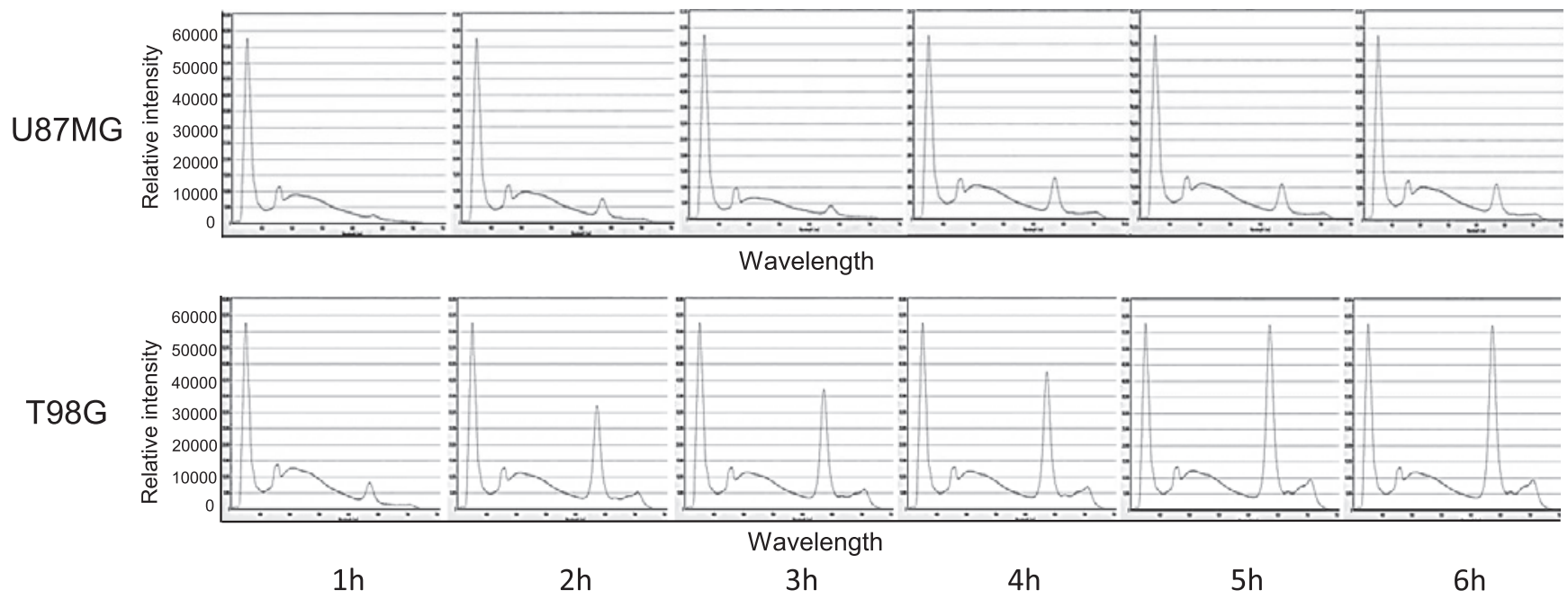

Fig. 3 PpIX level in glioma cell lines.

PpIX levels at 1 to 6 hours after 5-ALA administration are shown. The peak at a wavelength of 636 nm shows relative PpIX level. There was time-dependent intracellular accumulation of PpIX in glioma cell lines after exogenous 5-ALA administration. PpIX accumulation differed in relation to cell line. 


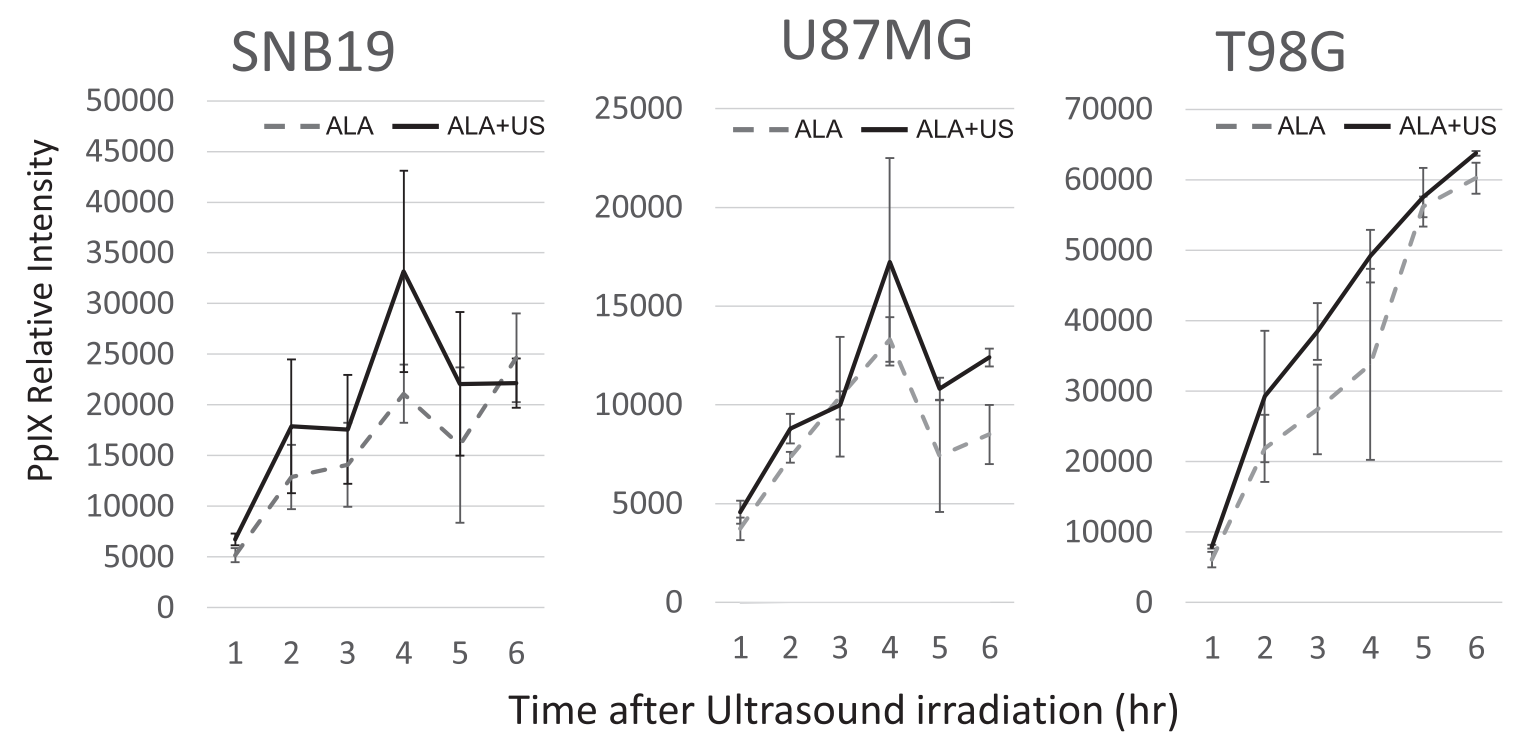

Fig. 4 Changes in PpIX level of ALA and ALA+US glioma cells. ALA+US glioma cells showed higher PpIX levels in all cell lines (mean $\pm S E, n=3$ ).

PpIX relative intensity in the ALA+US group reached a peak at 4 hours after ultrasound irradiation in the SNB19 and U87MG cell lines (Fig. 4). PpIX expression in T98G continued to increase until 6 hours after ultrasound exposure. In all cell lines, PpIX expression was higher than the level after 5-ALA administration without ultrasound at all time points, except for SNB19 after 6 hours' incubation.
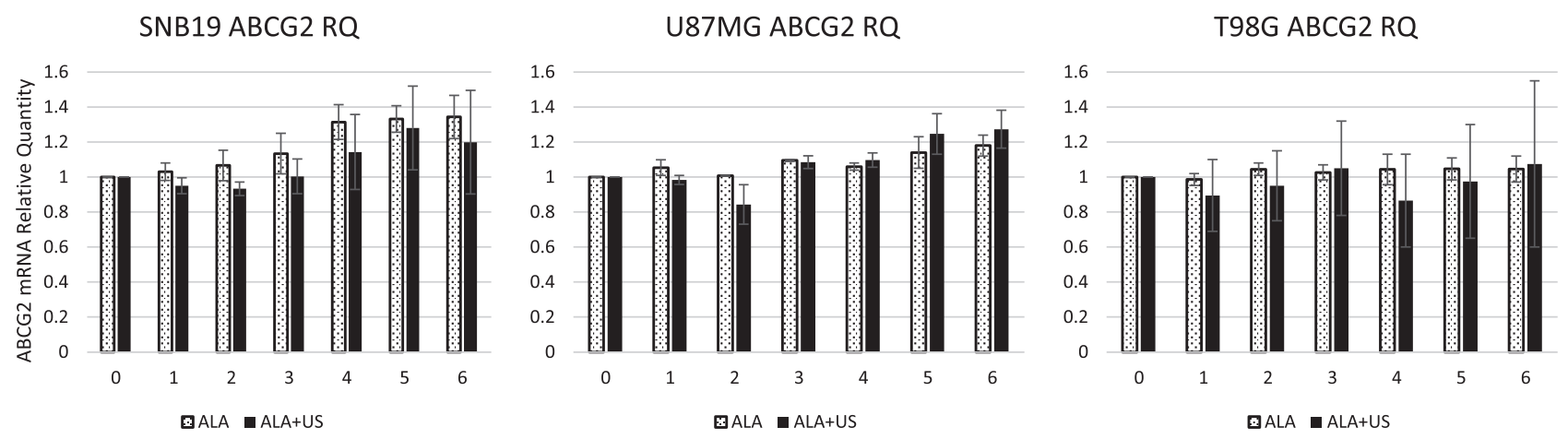

Fig. 5 Relative mRNA expression levels of ABCG2 after 5-ALA administration

The glioma cell lines SNB19 and U87MG showed a time-dependent increase in ABCG2 mRNA. However, ABCG2 expressions of T98G glioma cells did not change during observation. Ultrasound irradiation suppressed the increase in ABCG2 expression induced by ALA exposure in all three cell lines. The suppressive effect was seen for 6 hours in SNB19 cells and persisted for 2 hours after ultrasound irradiation in U87MG and T98G cells. Within 5 hours after ultrasound irradiation, this effect gradually diminished, and ABCG2 mRNA levels increased to the level of the non-ultrasound condition in T98G cells at 6 hours after US irradiation. In U87MG cells, ABCG2 expression was greater than that in the ALA only group after 4 hours (mean $\pm S E, n=3)$.

However, no cell death was observed, and floating cells reattached to the dish floor within 6 hours after ultrasound treatment. The ultrasound conditions of these experiments were not cytocidal for malignant gliomas.

\section{Variation of PpIX Accumulation in Cell Lines}

PpIX levels gradually increased after ALA administration. The peak level and timing differed in relation to cell line. SNB19, U87MG, and T98G cells peaked at 4, 4, and 6 hours after treatment, respectively. The relative level of PpIX in the same number of cells at 4 hours after 5-ALA administration was highest in T98G cells. We previously reported a difference in PpIX accumulation among cell lines $^{15}$. This difference suggests that the rate of PpIX production induced from exogenous 5-ALA differs. A key factor is ferrochelatase (FECH), which converts PpIX to heme and is diminished or absent in cancer cells. In addition, recent studies suggest that ABCG2 is crucial in regulating cellular accumulation of porphyrins in cancer cells ${ }^{16}$. 


\section{Increase in ABCG2 after ALA Administration}

PpIX levels are regulated in many cell types, because excess PpIX can undergo the iron-catalyzed Fenton reaction, which may generate potential DNA damage by reactive oxygen species (ROS) $)^{17}$. In the porphyrin synthetic and metabolic pathway, porphyrin transporters such as ABCB6 and ABCG2 are believed to be important in maintaining a homeostatic level of porphyrins. Increased ABCG2 activity decreased the intracellular PpIX level after ALA administration ${ }^{18}$ and reduced PpIX fluorescence ${ }^{19}$.

In our study, after ALA administration, ABCG2 expression slightly increased over time in glioma cell lines. Exogenous ALA yields a high production rate of porphyrin derivatives, including PpIX, in the porphyrin synthetic pathway. This elevation of porphyrins is thought to upregulate the activity of membrane transporters such as ABCG2 in expelling porphyrins to protect cells from porphyrin overaccumulation ${ }^{20}$. This was observed in glioma cells in our experiments.

\section{Effect of Ultrasound on PpIX Elevation}

Ultrasound has a variety of biological effects, including cell lysis, changes in cell division capability, ultrastructural changes, chromosomal and cytogenetic effects, and functional changes ${ }^{21}$. The effects of ultrasound on cells are classified as "gross effects", such as lysis, effects on cell division capability, and damage to cellular ultrastructure, and "subtle effects", such as chromosomal changes, functional changes, and altered growth patterns ${ }^{21}$. Among these, the most hazardous effect on cells is cell lysis; however, the conditions in our experiments $-1 \mathrm{MHz}, 3$ $\mathrm{W} / \mathrm{cm}^{2}$, and a duty cycle of $10 \%$-were below the threshold for such cell damage. We previously found no potential cell lysis that resulted in complete cellular disruption $^{15}$. The "subtle effects" of ultrasound may cause epigenetic changes, such as modification of histone protein structure, which can induce long-term effects on gene expression $^{21}$. Ultrasound may also lead to stimulation of cellular functions that mostly involve interactions at the cell membrane level ${ }^{21}$. In addition to membrane and mitochondrial damage caused by cavitation, Harvey et al. ${ }^{22}$ observed dilated rough endoplasmic reticulum and some irregular lesions. Generally, the cell nucleus seems unaffected by ultrasound exposure ${ }^{23,24}$. These effects of ultrasound suggest that various factors might affect enzymes and transporters in the porphyrin synthetic and metabolic pathways. These alterations might have induced early and higher PpIX expression, perhaps by accelerating 5-ALA incorporation by upregulation of PEPT1 or PEPT2 and accumulation of PpIX by suppression of
FECH or ABCG2. However, the biomechanism underlying the change in PpIX expression pattern is not well understood.

\section{Inhibition of ABCG2 by Ultrasound Irradiation}

Guo et al. $^{7}$ recently reported that ultrasound reversed chemoresistance in breast cancer stem-like cells by altering ABCG2 expression. In our study, we investigated the effect of ultrasound on glioma cells in relation to ABCG2 expression and found a similar response of glioma cells to ultrasound. ABCG2 is generally located in the cellular plasma membrane and is known as a porphyrin efflux pump, which exports harmful toxins and xenobiotics, such as potentially toxic porphyrins, from the intracellular to the extracellular environment'. Importantly, ABCG2 has a high affinity for porphyrins, i.e., hematoporphyrin and pheophorbide $\mathrm{a}^{25}$ and transports them in an ATPdependent manner ${ }^{25-27}$. ABCG2 is also located in mitochondrial membranes and contributes to PpIX efflux from mitochondria ${ }^{28}$.

Tyrosine kinase inhibitors such as imatinib mesylate and gefitinib are reported to inhibit ABCG2, which enhances the efficacy of photodynamic therapy by increasing PpIX level ${ }^{29,30}$. However, these chemotherapeutic agents are not suitable for enhancing photodiagnosis, because dosage, delivery, and timing cannot be easily controlled. In contrast, ultrasound is a mechanical effect that differs from drugs administered into the body. Conditions such as power, duration, and application site can be decided according to the intended purpose. We found that ultrasound suppressed ABCG2 expression in glioma cells against the increase in ABCG2 mRNA levels after 5ALA administration (Fig. 5). Since the knockdown of ABCG2 expression by siRNA is reported to increase accumulation of PpIX in pancreatic cancer cells ${ }^{31}$, this effect of ultrasound on ABCG2 is also believed to contribute to increasing PpIX in glioma cells.

\section{Limitations}

In this study, the ultrasound irradiation conditions were identical to those used in our previous study ${ }^{15}$. The ideal conditions would be optimized to best accelerate fluorescence intensity. Further research is necessary to identify the conditions that maximize both PpIX accumulation in tumor cells and patient safety.

The effect of ultrasound on non-tumorous cells should be considered in clinical settings. Further research is necessary to clarify the effects of ultrasound on normal cells, such as neurons, glial cells, and vascular endothelial cells. 


\section{Conclusion}

Ultrasound induced an early and accelerated increase in protoporphyrin IX level in glioma cells. Suppression of ABCG2 by ultrasound may contribute to PpIX accumulation after administration of 5-aminolevulinic acid to gliomas. Ultrasound irradiation before photodiagnosis may be feasible for early and enhanced detection of glioma.

Conflict of Interest: The authors have no conflicts of interest regarding this study.

\section{References}

1. Kaneko S. Photodynamic applications using aminolevulinic acid in neurosurgery. In: Okura I, Tanaka TE, editors. 5- Aminolevulinic acid science, technology and application: SBI ALAPROMO; 2011.

2. Austin Doyle L, Yang W, Abruzzo LV, et al. A multidrug resistance transporter from human MCF-7 breast cancer cells. Proc Natl Acad Sci U S A. 1998;95(26):15665-70.

3. Stummer W, Reulen HJ, Novotny A, Stepp H, Tonn JC. Fluorescence-guided resections of malignant gliomas - An overview. Acta Neurochir Suppl [Internet]. 2003 [cited 2019 Nov 11];88:9-12. Available from: http://www.ncbi.nl m.nih.gov/pubmed/14531555

4. Wakabayashi K, Nakagawa H, Adachi T, et al. Identification of cysteine residues critically involved in homodimer formation and protein expression of human ATP-binding cassette transporter ABCG2: A new approach using the Flp recombinase system. J Exp Ther Oncol. 2006;5(3):20522.

5. Wakabayashi K, Nakagawa H, Tamura A, et al. Intramolecular disulfide bond is a critical check point determining degradative fates of ATP-binding cassette (ABC) transporter ABCG2 protein. J Biol Chem [Internet]. 2007 Sep 21 [cited 2019 Nov 11];282(38):27841-6. Available from: http:/ /www.ncbi.nlm.nih.gov/pubmed/17686774

6. Jonker JW, Buitelaar M, Wagenaar E, et al. The breast cancer resistance protein protects against a major chlorophyll-derived dietary phototoxin and protoporphyria. Proc Natl Acad Sci U S A. 2002;99(24):15649-54.

7. Guo L, Zheng P, Fan $\mathrm{H}$, et al. Ultrasound reverses chemoresistance in breast cancer stem cell like cells by altering ABCG2 expression. Biosci Rep. 2017;37(6):1-11.

8. Zhou S, Zong Y, Ney PA, et al. Increased expression of the Abcg2 transporter during erythroid maturation plays a role in decreasing cellular protoporphyrin IX levels. Blood [Internet]. 2005 [cited 2019 Nov 4];105(6):2571-6. Available from: https://neo.nms.ac.jp/pubmed/,DanaInf $\mathrm{o}=$ www.ncbi.nlm.nih.gov,SSL+?term $=$ Blood $+2005 \% 3 \mathrm{~B} 10$ $5 \% 3 \mathrm{~A} 2571-6++$ Zhou $+\mathrm{S} \% 2 \mathrm{C}+\mathrm{Z}$ ong $+\mathrm{Y} \% 2 \mathrm{C}+\mathrm{Ney}+\mathrm{PA} \% 2 \mathrm{C}+$ Nair $+\mathrm{G} \% 2 \mathrm{C}+$ Stewart+CF\%2C+Sorrentino+BP

9. Krishnamurthy P, Ross DD, Nakanishi T, et al. The stem cell marker Bcrp/ABCG2 enhances hypoxic cell survival through interactions with heme. J Biol Chem. 2004 Jun 4; 279(23):24218-25.

10. Tamura A, An R, Hagiya $Y$, et al. Drug-induced phototoxicity evoked by inhibition of human $\mathrm{ABC}$ transporter ABCG2: Development of in vitro high-speed screening systems. Expert Opin Drug Metab Toxicol. 2008 Mar;4(3): $255-72$.
11. Nadar SS, Rathod VK. Ultrasound assisted intensification of enzyme activity and its properties: a mini-review. World J Microbiol Biotechnol [Internet]. 2017 Aug 22 [cited 2019 Nov 7];33(9):170. Available from: http:/ /www. ncbi.nlm.nih.gov/pubmed/28831716

12. Wang $\mathrm{Y}$, Wang $\mathrm{W}, \mathrm{Xu} \mathrm{H}$, et al. Non-lethal sonodynamic therapy inhibits atherosclerotic plaque progression in ApoE - / - mice and attenuates ox-LDL-mediated macrophage impairment by inducing heme oxygenase-1. Cell Physiol Biochem. 2017;41(6):2432-46.

13. Gross JL, Behrens DY, Mullins DE, Kornblith PL, Dexter DL. Plasminogen activator and inhibitor activity in human glioma cells and modulation by sodium butyrate. Cancer Res [Internet]. 1988 [cited 2019 Nov 4];48(2):291-6. Available from: https://neo.nms.ac.jp/pubmed/,DanaInf o=www.ncbi.nlm.nih.gov,SSL+3121170

14. Eléouet S, Rousset N, Carré J, et al. In vitro fluorescence, toxicity and phototoxicity induced by $\delta$-aminolevulinic acid (ALA) or ALA-esters. Photochem Photobiol. 2000;71 (4): 447 .

15. Ju D, Yamaguchi F, Zhan G, et al. Hyperthermotherapy enhances antitumor effect of 5-aminolevulinic acidmediated sonodynamic therapy with activation of caspase-dependent apoptotic pathway in human glioma. Tumor Biol [Internet]. 2016. Available from: http://link.sp ringer.com/10.1007/s13277-016-4931-3

16. Ishikawa $\mathrm{T}$, Takahashi $\mathrm{K}$, Ikeda $\mathrm{N}$, et al. Transportermediated drug interaction strategy for 5-aminolevulinic acid (ALA)-based photodynamic diagnosis of malignant brain tumor: Molecular design of ABCG2 inhibitors. Pharmaceutics. 2011;3(3):615-35.

17. Krishnamurthy $\mathrm{P}, \mathrm{Xie} \mathrm{T}, \mathrm{Schuetz}$ JD. The role of transporters in cellular heme and porphyrin homeostasis. Pharmacol Ther. 2007;114(3):345-58.

18. Robey RW, Steadman K, Polgar O, Bates SE. ABCG2mediated transport of photosensitizers: Potential impact on photodynamic therapy. Cancer Biol Ther. 2005 Feb;4 (2):187-94.

19. Barron GA, Moseley H, Woods JA. Differential sensitivity in cell lines to photodynamic therapy in combination with ABCG2 inhibition. J Photochem Photobiol B Biol. 2013;126:87-96.

20. Krishnamurthy P, Schuetz JD. The role of ABCG2 and ABCB6 in porphyrin metabolism and cell survival. Curr Pharm Biotechnol. 2011;12(4):647-55.

21. Health Protection Agency. Health effects of exposure to ultrasound and infrasound. Report of the independent advisory group on non-ionising radiation [Internet]. 2010. Available from: http://www.hpa.org.uk/web/HPAweb\& HPAwebStandard/HPAweb_C/1265028759717

22. Harvey W, Dyson M, Pond JB, Grahame R. The "in vitro" stimulation of protein synthesis in human fibroblasts by therapeutic levels of ultrasound. Rheumatol Rehabil. 1975;14(4):237.

23. Ter Haar G, Dyson M, Smith SP. Ultrastructural changes in the mouse uterus brought about by ultrasonic irradiation at therapeutic intensities in standing wave fields. Ultrasound Med Biol. 1979;5(2):167-79.

24. Watmough DJ, Dendy PP, Eastwood LM, et al. The biophysical effects of therapeutic ultrasound on HeLa cells. Ultrasound Med Biol. 1977;3(2-3):205-19.

25. Tamura A, Onishi $Y, A n R$, et al. In vitro evaluation of photosensitivity risk related to genetic polymorphisms of human ABC transporter ABCG2 and inhibition by drugs. Drug Metab Pharmacokinet [Internet]. 2007 Dec [cited 2019 Nov 11];22(6):428-40. Available from: http://www.n 
cbi.nlm.nih.gov/pubmed/18159130

26. Tamura A, Watanabe $\mathrm{M}$, Saito $\mathrm{H}$, et al. Functional validation of the genetic polymorphisms of human ATP-binding cassette $(\mathrm{ABC})$ transporter ABCG2: Identification of alleles that are defective in porphyrin transport. Mol Pharmacol. 2006;70(1):287-96.

27. An R, Hagiya Y, Tamura A, et al. Cellular phototoxicity evoked through the inhibition of human $A B C$ transporter ABCG2 by cyclin-dependent kinase inhibitors in vitro. Pharm Res. 2009 Feb;26(2):449-58.

28. Kobuchi H, Moriya K, Ogino T, et al. Mitochondrial localization of $\mathrm{ABC}$ transporter ABCG2 and its function in 5aminolevulinic acid-mediated protoporphyrin IX Accumulation. PLoS One. 2012;7(11):e50082.

29. Liu W, Baer MR, Bowman MJ, et al. The tyrosine kinase inhibitor imatinib mesylate enhances the efficacy of photodynamic therapy by inhibiting ABCG2. Clin Cancer Res [Internet]. 2007 Apr 15 [cited 2019 Nov 5];13(8):2463-70. Available from: http://www.ncbi.nlm.nih.gov/pubmed/1 7438106

30. Sun W, Kajimoto $\mathrm{Y}$, Inoue $\mathrm{H}$, et al. Gefitinib enhances the efficacy of photodynamic therapy using 5-aminolevulinic acid in malignant brain tumor cells. Photodiagnosis Photodyn Ther. 2013 Feb;10(1):42-50.

31. Kawai N, Hirohashi Y, Ebihara Y, et al. ABCG2 expression is related to low 5-ALA photodynamic diagnosis (PDD) efficacy and cancer stem cell phenotype, and suppression of ABCG2 improves the efficacy of PDD. PLoS One. 2019; 14(5):1-15.

(Received, January 17, 2020)

(Accepted, January 24, 2020)

(J-STAGE Advance Publication, March 31, 2020)

Journal of Nippon Medical School has adopted the Creative Commons Attribution-NonCommercial-NoDerivatives 4.0 International License (https://creativecommons.org/licenses/by-nc-nd/4.0/) for this article. The Medical Association of Nippon Medical School remains the copyright holder of all articles. Anyone may download, reuse, copy, reprint, or distribute articles for non-profit purposes under this license, on condition that the authors of the articles are properly credited. 